\title{
LA REVALORIZACIÓN DE ÁMBITOS Y SABERES LOCALES COMO FACTORES ESTRATÉGICOS EN LA CONSECUCIÓN DE UN HÁBITAT DIGNO
}

\section{THE REVALORIZATION OF LOCAL AREAS AND LOCAL KNOWLEDGE AS STRATEGIC FACTORS IN THE ACHIEVEMENT OF A DECENT HABITAT}

Daniela Gargantini ${ }^{1}$

Noelia Cejas $^{2}$

\section{RESUMEN}

La problemática habitacional resulta una deuda pendiente a nivel nacional y regional. Aún frente a fuertes procesos de inclusión de la misma en las agendas gubernamentales, los porcentajes deficitarios de hogares que no acceden a un hábitat digno no ha disminuido en las últimas décadas, consolidando porcentajes históricos. Dichos resultados parecen estar vinculados a los modos con que se ha intentado abordar la problemática antes que a la disposición de recursos decisionales y materiales.

Frente a este marco contextual, el presente artículo busca problematizar la perspectiva reductiva con que el Estado aborda las problemáticas habitacionales, y presenta dos perspectivas orientadas a complejizar ese enfoque, plausibles de ser complementarias entre sí, particularmente referidas a la valorización de los saberes y ámbitos locales como actores estratégicos en la generación de soluciones para la consecución de un hábitat digno.

El artículo se organiza a partir de dos niveles conceptuales, que permiten, a partir de su articulación, desarrollar campos de sentido y nociones específicas en torno a la dimensión política de la producción de hábitat. Félix Guattari y Gilles Deleuze proponen abordar los procesos sociales desde

\footnotetext{
${ }^{1}$ Doctora en Arquitectura y Pos-Doctora de la Universidad Nacional de Rosario (Argentina), investigadora adjunta del Consejo Nacional de Investigaciones Científicas y Tecnológicas en el Centro Experimental de la Vivienda Económica. Docente-investigadora de la Universidad Católica de Córdoba. Docente en posgrados tanto de América Latina como de Europa. Línea de investigación actual: acceso al hábitat en municipios, condiciones y capacidades institucionales para la gestión local e integral del hábitat. Datos laborales: Igualdad 3585- Villa Siburu (5000) Córdoba, Argentina. Tel 0054351 48944442/ Datos personales: Rafael Núñez 62 (2434) Arroyito, Córdoba, Argentina. Tel 00549351 114229. dmgargantini@gmail.com

${ }^{2}$ Doctora en Estudios Sociales de América Latina y Licenciada en Comunicación Social por la Universidad Nacional de Córdoba (Argentina), investigadora asistente del Consejo Nacional de Investigaciones Científicas y Tecnológicas en el Centro Experimental de la Vivienda Económica. Línea de investigación actual: Diálogo de saberes para el desarrollo de tecnología social en el campo del hábitat. Datos laborales: Igualdad 3585- Villa Siburu (5000) Córdoba, Argentina. Tel 0054351 4894442/ Datos personales: Ángel Monasterio 3823 (5002) Córdoba, Córdoba, Argentina. Tel 00549351 3638793.noelia_cejas@outlook.com
} 
pares de categorías (no oposicionales), entre las que el artículo recupera las nociones de micro y macropolítica, y sus sentidos molar y molecular. El desarrollo de estas nociones permite desplegar redes de conceptos específicos para cada nivel de observación.

Se busca con ello nutrir la reflexión y el debate en la temática en pos de brindar soportes conceptuales para el reconocimiento de condiciones y capacidades a impulsar en pos del diseño, gestión e implementación de soluciones más pertinentes y efectivas en el campo habitacional.

PALABRAS CLAVE : Hábitat - Micropolítica - Macropolítica - Estado - Territorio

\section{ABSTRACT}

The housing problem is a pending debt at national and regional level. Even in the face of processes of inclusion in government agendas, the deficit percentages of households that do not have access to a decent habitat have not decreased in the last decades, consolidating historical percentages. These results seem to be linked to the ways in which the problem has been addressed rather than to the provision of decision and material resources.

Faced with this contextual framework, this article seeks to problematize the reductive perspective with which the State approaches housing problems. Two perspectives are presented aimed at making this approach more complex, plausible to be complementary to each other, particularly referring to the valorisation of knowledge and local spheres as strategic actors in the generation of solutions for the achievement of a decent habitat.

The article is organized from two conceptual levels, which allow, from its articulation, develop fields of meaning and specific notions around the political dimension of habitat production. Félix Guattari and Gilles Deleuze propose to approach social processes from pairs of categories (non-oppositional), among which the article recovers the notions of micro and macropolitics, and their molecular and molar senses. The development of these notions allows the deployment of networks of specific concepts for each level of observation.

The aim is to broaden reflection and debate on the subject in order to provide conceptual supports for the recognition of conditions and capacities to be promoted in the design, management and implementation of more relevant and effective solutions in the field of housing.

KEYWORDS: Habitat - Micropolitics - Macropolitics - State - Territory 


\section{INTRODUCCIÓN}

La cuestión habitacional en toda la región constituye un factor ineludible en la determinación de las condiciones materiales del fenómeno de la pobreza. Junto con ello la experiencia del habitar, más allá de la escala, ubicación geográfica o nivel de interrelación existente con procesos globales del lugar que se habite, constituye un fenómeno esencialmente local.

Sin embargo, las políticas habitacionales -aun con algunas diferencias y excepciones- fuertemente ancladas en un enfoque clásico productor de viviendas y hechos físicos, ha desconocido esta dimensión, llegando a omitirla e incluso a anularla en sus intervenciones. La incapacidad de establecer vínculos conducentes a la producción de un enfoque integral ha contribuido a ello, y bajo este enfoque se han reproducido soluciones estandarizadas que no permitieron que prosperaran soluciones habitacionales adaptadas a las realidades locales. Así han resultado insuficientes e ineficientes no sólo cuantitativa sino cualitativamente (Autora, año).

Frente a esta persistencia, desde diversas perspectivas teóricas se han venido problematizando algunos aspectos que parecieran prefigurarse como estratégicos a la hora de pensar soluciones más pertinentes, los cuales se encuentran relacionados a la incapacidad de recuperar actores y saberes locales.

En ese sentido, en este texto nos interesa realizar un trabajo de articulación conceptual que permita complejizar algunas perspectivas parciales que se desarrollan en el campo del hábitat, sin por ello pretender dar cuenta de la totalidad de los fenómenos que lo componen. Metodológicamente esto supone una tarea eminentemente teórica, orientada a recuperar aportes significativos de distintos autores. Cabe decir que las "regularidades empíricas" (Sautu y otros, 2005:42), emergentes de distintas experiencias de investigación en las cuales las autoras de este artículo se insertan, constituyen la perspectiva privilegiada para desplegar la articulación a desarrollar.

Como categorías estructurantes u organizadoras del planteo, este artículo recupera aportes conceptuales acuñados por Deleuze y Guattari ${ }^{1}$, particularmente los de macropolítica y micropolítica. Se trata de conceptualizaciones densas, difíciles de demarcar en sus límites y, tal vez por ello, profundamente potentes. El nivel macropolítico, articulado con la idea de lo molar, refiere a los espacios instituidos de la política, aquellos que han cristalizado en instituciones tales como el Estado, los tribunales, los parlamentos. En otro nivel, solo distinguible en un sentido analítico, proponen el concepto de micropolítica. Con ello remiten a los campos de producción de sentido que 
no han cristalizado en instituciones, pero que pugnan (aunque no violentamente) por manifestarse, sostenerse o fortalecerse. El nivel de la micropolítica procura reconocer los modos de reproducción y/o transformación de los modos de producción de subjetividad dominantes. En definitiva, se trata de una perspectiva de análisis que integra niveles de mayor concreción, como pueden ser las propias organizaciones y sus normativas, hasta los niveles más intangibles como puede ser el proceso de construcción de subjetividad, entendida como aquello que compone las matrices de sentido de los sujetos: mecanismos de percepción, de afectividad, de producción de conocimiento, de memoria, etc.

Bajo este enfoque amplio, podemos integrar aportes que provienen desde el institucionalismo y la administración pública, a fin de profundizar en el nivel macropolítico de la producción de hábitat. Desde estas perspectivas se ha revalorizado el reconocimiento de la necesaria articulación con los gobiernos locales como nivel del Estado que posee mayores incumbencias y competencias en el manejo de lo habitacional como elemento estructurante de las políticas urbano-territoriales. "En este marco, las actuales políticas de vivienda y de desarrollo urbano local de las ciudades latinoamericanas deben ser redefinidas para adoptar un enfoque de derechos humanos, de derecho a la vivienda y a la ciudad, adoptando criterios de sustentabilidad económica, social y ambiental" (Ziccardi y Cravacuore, 2017: 5-6). En este proceso se revaloriza la incipiente y no lineal incorporación de estrategias y abordajes contextualizados, integrales y multiactorales, presentes en los procesos de coexistencia y convivencia diaria con modelos clásicos en el campo local. Son éstos los que a partir de una constante tensión, confrontación y puja van fortaleciendo -desde los modelos híbridos vigentes- puntos de entrada viables o hendijas de flexibilización en las políticas habitacionales instituidas (Autora, año).

Por su parte, desde una perspectiva micropolítica, el trabajo busca preguntarse por los efectos de posibilidad que otorgan las intervenciones materiales provistas por el Estado, y abrir allí un cuestionamiento a los mecanismos de articulación con saberes, memorias y sensibilidades propias de las comunidades a las que se dirigen las políticas habitacionales. Estos habilitan un espacio de diálogo con las comunidades objeto de estas iniciativas, entendidos también como actores estratégicos en el diseño, gestión y desarrollo de políticas habitacionales. En ese sentido, se recuperan conceptualizaciones que profundizan en la dimensión política de la tecnología, señalando la no neutralidad de la misma, y enfoques sociológicos que permiten pensar el diálogo de saberes, como estrategia superadora de enfoques verticalistas, transferencistas y asistencialistas (Autora, año). 
Ambas perspectivas colocan en el centro de la escena el debate sobre la concepción e inclusión de "lo local" en el diseño, gestión e implementación de las políticas habitacionales, y de su importancia a la hora de pensar soluciones más pertinentes.

Desde los aportes provenientes del desarrollo local y según González Meyer, entendemos a lo local como

"una escala de lo social (Arocena, 1995) y como una producción social (González, 2006); es decir, cuya dinámica resulta de la interacción de múltiples agentes. Estos agentes producen lo local actuando desde diversas escalas de la realidad - local, regional, nacional, global-, desde diversas esferas de la realidad-económica, política, cultural-y desde diferentes tipos de relaciones entre ellos, ya sea de oposición, negociación o cooperación." (González Meyer 2009: 69)

Recuperando esta definición, interesa ponderar una perspectiva de lo local que subraya la multiplicidad de aristas, lo cual caracteriza un escenario socialmente denso e intrínsecamente articulado con los diferentes niveles políticos, micro y macro. De hecho, Gonzalez Mayer (2009: $69,71,74)$ va a señalar la concepción genérica de lo local se asimila a "a la idea de espacios subnacionales (...) que constituyen un meso-nivel [y] resultan importantes para efectos de estrategias más endógenas de desarrollo." Así, se considera relevante la "articulación de lo social con lo geográfico-físico", donde "la dimensión identitaria es expresada en una historia particular y en un territorio limitado" (Arocena, 1995), constituyéndose así en "sujeto político de las trayectorias de desarrollo"

Bajo esta concepción y sin ignorar la existencia de ciertos poderes periféricos que actúan en y desde los territorios locales (Grémion, 1996), resulta importante reconocer y valorar a los "agentes propiamente locales -es decir, para quienes la sociedad local constituye su medio de vida, trabajo, reproducción y sentido de pertenencia principal-" (González Meyer, 2009: 70), impulsando propuestas que busquen hacer aumentar el peso que dichos agentes propiamente locales tienen sobre los procesos territoriales subnacionales y propiciando perspectivas más endógenas de análisis e ideario que descubran y proyecten la fuerza real y potencial de este tipo de agentes.

En este sentido, renunciando a una mirada ingenua e idealista que desconozca las tensiones, conflictos y dificultades que pueden existir a nivel local, y considerando la fuerza constituyente que también tienen los agentes supralocales, las redes globales y las interdependencias socio-espaciales, "mirar la realidad desde lo local, con centralidad en los agentes locales que son parte de la producción de las realidades territoriales puede ayudar a visibilizar y empujar procesos políticos liberadores, ampliando los campos de acción de sectores sociales locales subalternos" (González 
Meyer, 2009: 75), dado que es en estos ámbitos locales, reconocidos como productos sociales y objetos de la política, donde se define significativamente el bienestar personal y colectivo de las personas que los habitan.

Este enfoque que se postula

"no significa retrotraer la mirada solo a lo local, haciendo apología de esa escala de mirada y de vida, sino reconocer que desde todas las escalas -desde lo local a lo global- la mirada observa y descubre aspectos de la totalidad no visibles desde la otras. La construcción de lo social, por tanto, necesita, por tanto, ese juego de racionalidades y acciones múltiples, que es realizada por agentes concretos, en cuanto a escalas y niveles de la realidad. Es en esa tensión no eliminable que la revalorización de lo local contribuye a mostrar realidades y potencialidades sociales, ocultas y ocultadas" (González Meyer, 2009: 77-78),

y que resultan estratégicas a la hora de pensar los procesos de acceso al hábitat.

Desde esta concepción sobre lo local, este trabajo busca nutrir la reflexión y el debate en la temática en pos de brindar soportes conceptuales para el reconocimiento de condiciones y capacidades a impulsar en pos del diseño, gestión e implementación de soluciones habitacionales más pertinentes y efectivas.

\section{LOS APORTES DE GUATTARI Y DELEUZE EN LA DEFINICIÓN DE CATEGORÍAS ANALÍTICAS}

Los autores Gilles Deleuze y Félix Guattari (1997) articulan discusiones de la filosofía, el psicoanálisis y la semiótica, en una genealogía que integra a Spinoza, Nietzsche, Marx, Freud, entre otros. En su trabajo subyace una preocupación constante por las relaciones de poder y en efecto desarrollan un sistema de categorías que permiten abordar la complejidad del fenómeno.

En ese sentido, los conceptos de macro y micropolítica, tanto como los conceptos de molarmolecular, constituyen dispositivos de análisis potentes, cuya principal virtud es la de poder ingresar a distintos niveles de observación sin producir relaciones de oposición binaria entre estos. En uno de sus textos más reconocidos, "Mil Mesetas: capitalismo y esquizofrenia" (1997), Deleuze y Guattari señalan que "toda política es a la vez macropolítica y micropolítica" (Deleuze, G., Guattari, F., 1997: 83). Esta afirmación, lejos de simplificar o vaciar de contenido las discusiones sobre la dimensión de lo político en lo social, habilitan nuevas y más profundas herramientas de análisis:

"Contra ese tipo de afirmación se acostumbra usar el famoso argumento «si la política está por todas partes, no está en ninguna» [...] respondería que 
efectivamente la política y la micropolítica no están en todas partes y que la cuestión es justamente la de colocar la micropolítica en todas partes -en nuestras relaciones estereotipadas de la vida personal, de la vida conyugal, de la vida amorosa y de la vida profesional, en las cuales todo es guiado por códigos. Se trata de hacer entrar en todos esos campos un nuevo tipo de pragmática: un nuevo tipo de análisis que corresponda de hecho a un nuevo tipo de política" (Guattari y Rolnik, 2013: 157)

Esta perspectiva, que reconoce los espacios "tradicionales" de la política al mismo tiempo que traza una relación de conjunto con nuevas aristas de problematización, abrió un espacio de debate sumamente fértil, que dialoga con trabajos de otros autores. Se destacan los aportes de Foucault quien despliega este enfoque en el concepto de biopolítica- y también la perspectiva feminista de finales de los años 60 y principios de los 70, cuyo movimiento construyó un estandarte con "Io personal es político", a fin de visibilizar la politicidad de los espacios privados.

Si la macropolítica hace referencia a la política que se produce en y desde los espacios tradicionales para la misma (esto es: el Estado y sus esferas de gobierno, los tribunales, parlamentos), la noción de micropolítica hace referencia al carácter igualmente político de espacios de producción de sentido antes invisibilizados. Se inscriben en este último toda una serie de contextos que desde los años sesenta cobran visibilidad, otrora excluidos de la política, por su supuesta pertenencia a la esfera de lo privado: las relaciones sexuales, familiares, residenciales, laborales, institucionales, clínicas o escolares.

Para este trabajo, interesa ingresar a la escala macro y micropolítica de aquello que puede ser comprendido como lo local en procesos de producción de hábitat. De esta manera es posible comprender los espacios institucionales desde los cuales se configura un tipo de comprensión sobre la producción de hábitat, en cuyo despliegue se identifican dispositivos orientados a intervenir en municipios y comunidades y, al mismo tiempo, es posible comprender los procesos de producción de hábitat en la escala no sólo de los sujetos, habitantes, sino también de los propios gobiernos locales, considerados ambos como simples destinatarios de las políticas habitacionales. En ese sentido es posible discutir la pasividad con que se define y concibe, bajo la lógica de "destinatarios", a quienes habitan o simplemente ejecutan políticas definidas desde ámbitos de mayor nivel.

Estos dos niveles de lo local, macro y micropolítico, no constituyen territorialidades estáticas; por el contrario, se despliegan en dinámicas singulares, atravesamientos, que interesa analizar. Esto da lugar para presentar otro par conceptual a recuperar aquí: lo molar-molecular. Guattari va a señalar que no existe lógica de contradicción entre los niveles molar y molecular:

"Las mismas especies de elementos, los mismos tipos de componentes individuales y colectivos en juego en un determinado espacio social pueden 
funcionar de modo emancipador a nivel molar $y$, extensivamente, ser extremadamente reaccionarios a nivel molecular. La cuestión micropolítica es la de cómo reproducimos (o no) los modos de subjetivación dominantes. Así, por ejemplo, un grupo de trabajo comunitario puede tener una acción nitidamente emancipadora a nivel molar, y al mismo tiempo a nivel molecular puede tener toda una serie de mecanismos de liderazgo falocráticos, reaccionarios, etc. [...] Oponer una política molar de las grandes organizaciones, presentes en cualquier nivel de la sociedad (micro o macro), a una función molecular que considera las problemáticas de la economía del deseo, igualmente presentes en cualquier nivel de la sociedad, no implica una apreciación en la cual lo molecular sería lo bueno y lo molar, lo malo. Los problemas se plantean siempre y al mismo tiempo en los dos niveles". (Guattari y Rolnik, 2013:155)

Así, el nivel micropolítico aborda la producción subjetiva, reconociendo atravesamientos molares y moleculares. Guattari plantea que la producción de subjetividad se da en procesos doblemente descentrados del sujeto, donde no es posible contraer este proceso a la escala de un individuo. Sostiene que se trata de un proceso que ocurre en una multiplicidad de agenciamientos sociales, entre los que se pueden distinguir mecanismos descentrados extrapersonales (sistemas económicos, sociales, tecnológicas, icónicos, de comunicación de masas, etc.) o infrapersonales (sistemas de percepción, de sensibilidad, de afecto, de deseo, de representación, de memoria, de producción de conocimiento, sistemas corporales, orgánicos, biológico, etc.) (Guattari y Rolnik, 2013). “El modo por el cual los individuos viven esa subjetividad oscila entre dos extremos: una relación de alienación y opresión, en la cual el individuo se somete a la subjetividad tal como la recibe, o una relación de expresión de la subjetividad, produciendo un proceso [...] de singularización". Guattari y Rolnik, 2013:48).

El interjuego de las categorías presentadas nos permitirá reconocer en el nivel macropolítico aquellos dispositivos institucionales que pugnan por recuperar y dinamizar los recursos, estrategias y conocimientos propios de las reparticiones locales, reconociendo en esta escala de gobierno la capacidad de leer con mayor detenimiento las necesidades y potencialidades de su territorio. Asimismo, en el nivel micropolítico, intentaremos reconocer los juegos de producción y reproducción de los sentidos que los actores despliegan, en el marco de las intervenciones estatales orientadas a resolver problemáticas de hábitat. Esto nos permitirá pensar a las tecnologías de hábitat como soportes discursivos, capaces de habilitar o inhibir agenciamientos por parte de los actores y ámbitos locales en las que se inscriben.

Con estas categorías nos proponemos realizar un análisis, tras el cual nos interesa señalar un espacio de interjuego molar-molecular entre niveles macro y micropolítico, capaz de producir aportes sustantivos a la comprensión y resolución de las problemáticas de hábitat, en que los ámbitos 
institucionales (macropolítica) de los gobiernos locales puedan habilitar mecanismos de apertura genuina al diálogo con actores de las comunidades, recuperando sus saberes y percepciones (micropolítica).

\section{LA DIMENSIÓN LOCAL DE LA MACROPOLÍTICA}

Tal como se ha expresado, la macropolítica hace referencia a la política que se produce en y desde los espacios tradicionales. Para el análisis de esta dimensión resulta útil establecer un diálogo conceptual con los aportes propuestos por Subirats et al (2008). En su esquema teórico, analiza variables e indicadores por fases de la política, estableciendo una clara diferenciación ente la fase de definición de la política (con sus sub-fases relacionadas al surgimiento de los problemas, incorporación en la agenda y definición política de los mismos) y la forma operativa o implementación de la política (relacionada a la formulación y decisión del programa, su implementación y evaluación), desde las cuales se pueden indagar variables propias a cada una o bien comunes a ambas.

Siguiendo este esquema analítico, el proceso de recentralización de las políticas en América Latina evidencia una recuperación del rol de los estados en algunas esferas (entre ellas las sociales) (Thwaites, 2010) pero a favor de los estados nacionales, recortando las competencias locales, desincentivando el fortalecimiento de la participación ciudadana y echando por tierra los esfuerzos por valorizar las realidades y saberes o capacidades locales, revirtiendo el empoderamiento municipal en las últimas tres décadas (Cravacuore, 2014 y 2015)

"Tras el mencionado proceso persiste por parte de los ámbitos centrales de gobierno la asignación real de un rol secundario, despolitizado, marginal, meramente operativo o ejecutor de políticas diseñadas en niveles superiores, sin mayor capacidad de incidencia para el gobierno local (Brugué y Gomà, 1998). (...) Explícitamente, refiriéndonos a la producción habitacional, la experiencia indica que el rol de los gobiernos locales en la fase de formulación de las políticas que la promueven es prácticamente nulo, si no marginal. Esto es reflejo de la desconfianza que los ámbitos de gobierno intermedio y central tienen para con los locales y que, según Brugué y Gomá (1998: 16), “... se traduce en limitaciones competenciales, poca generosidad en la transferencia de recursos, proliferación de mecanismos de control y tutela o restricciones legislativas a su capacidad de actuación". En la fase de implementación y gestión de las políticas mencionadas está presente de manera más visible la labor de los ámbitos de gobierno municipal, ya que son éstos quienes en general operan las acciones más concretas que se derivan de los programas y proyectos (Pedrotti, 2016) a través de "la adopción de roles meramente 
operativos", ejecutores de políticas diseñadas en ámbitos superiores (Brugué y Gomá 1998: 29)" (Autora, año, página)

Frente a esta tendencia y en consonancia con movimientos de alcance internacional se ha comenzado a promover la conciencia de los límites que este modelo de producción presenta, propiciando la puesta en marcha de dispositivos que favorecen resituar a los gobiernos y a las comunidades locales, para dar a estos ideales formas políticas concretas, transformándolos en realizaciones prácticas que los reconozcan y potencien (UCLGCGLU, 2016; Global Taskforce, 2016 y Naciones Unidas, 2016).

En materia de políticas habitacionales, a nivel macropolítico resulta estratégico la puesta en vigencia de algunas capacidades, entendidas como dispositivos con poder de potenciar trasformaciones en el sentido arriba descripto.

A nivel de definición de la política, destaca la consideración del municipio como principal nivel del estado responsable de brindar las condiciones para la resolución de las problemáticas existentes a nivel urbano-habitacional. En este sentido si bien algunas condiciones inhiben las capacidades y la autonomía político-financiera municipal, existen competencias otorgadas a este nivel específicamente relacionadas al desarrollo, la planificación y la intervención territorial-que pueden ser ejercidas y puestas en funcionamiento por los mismos gobiernos locales "con claro nivel de potencialidad a la hora de brindar soluciones a problemas de déficit habitacional, hábitats degradados y precios inaccesibles de los mercados inmobiliarios" (Petrelli, Galizzi y Orecchia, 2014: 29).

Junto con ello, la superación de una concepción del hábitat como simple resultado de productos construidos, conlleva a considerarlo como proceso social contextualizado y por lo mismo de carácter integral, multiactoral y local (Alcalá, 1995; Autora, año; Barreto y Alcalá, 2007; Naciones Unidas, 2016). De esta concepción se deriva el reconocimiento de que las necesidades habitacionales nunca son homogéneas (Rodríguez et al, 2011; Nirenberg et al, 2006), y por ende factibles de estandarización en sus soluciones, lo cual convoca a un conocimiento pormenorizado no sólo de las mismas sino particularmente de las capacidades disponibles y existentes en los sujetos, en el entorno y en todos los actores intervinientes en el territorio (sean estos locales o no).

Este conocimiento va acompañado de un reconocimiento de los diferentes sujetos y grupos sociales a poder definir sus necesidades, tanto como proponer y llevar adelante estrategias tendientes a su resolución, teniendo en consideración el acompañamiento particular a las diferentes trayectorias habitacionales y de vida de los hogares. 
La constitución de equipos interdisciplinarios y de mesas o espacios de articulación interactoral formalizadas que garanticen el involucramiento y la participación efectiva de los diferentes actores sociales en las distintas etapas del proceso habitacional (tanto entre diferentes niveles de gobierno (escala intergubernamental) como entre el Estado y diversos actores sociales (escala interinstitucional), con especial énfasis en la integración de la población participante, constituyen formas operativas que, a manera de hendijas de flexibilización, permiten introducir estrategias transformadoras y redistribuidoras de poder frente a los modelos hegemónicos y verticalistas de definición de los problemas a atender y las soluciones a otorgar (Ramírez, 2002).

Este modo de definir la política posee implicancias temporales, organizacionales y presupuestarias, dado que "las acciones habitacionales se conciben como procesos no solo técnicos sino sociopolíticos, lo que conlleva a renunciar a las iniciativas meramente simbólicas que facilitan la rápida visibilidad política y la legitimación coyuntural, por el desarrollo de iniciativas de acción efectiva y sostenible a largo plazo, que exceden los tiempos tradicionales de obra física" (Autora, año). De esta forma también se constituyen en estrategias de trasformación de la propia gestión estatal interna.

A nivel de la implementación de la política existen otras capacidades relacionadas a la formulación y decisión de los programas, su implementación y evaluación, necesarias para abrir espacios de reconocimiento de la micropolitica, capaces de producir aportes sustantivos a la comprensión y resolución de las problemáticas de hábitat.

Entre ellas destaca el necesario fortalecimiento del nivel de autonomía fiscal del municipio como forma de superar la tradicional subordinación a los fondos externos, que suele reducir los márgenes de actuación del gobierno local en materia urbano-habitacional. En este sentido el conocimiento y la utilización municipal de instrumentos de financiamiento y redistribución de costos y beneficios resultan estratégicos (Reese, 2003).

Junto con ello, el acceso al suelo urbano, el tipo de implantación territorial, las modalidades constructivas elegidas y los procesos potenciados, resultan elementos esenciales en la implementación de toda acción habitacional desde la perspectiva que este artículo postula.

El acceso al suelo resulta estratégico dado que posibilita un triple efecto: favorece una mejor y mayor integración de la política habitacional a procesos de crecimiento y consolidación urbana, habilita posibilidades para las familias de alcanzar un hábitat digno, y contribuye en otorgarles sentido de ciudadanía. En su resolución se destaca la necesidad de avanzar hacia una forma de concebir la 
propiedad bajo principios que prioricen los intereses colectivos por sobre los particulares y la potenciación de una mayor función reguladora del Estado.

Junto con ello, todo tipo de implantación territorial que promueva evitar la segmentación de los espacios urbanos, priorice la permanencia de la población en las zonas donde reside y rescate la opción de los actores sociales demandantes a ejercer la opción de elegir dónde y cómo vivir, es factor fortalecedor de la democracia y de la igualdad entre las personas, al mismo tiempo que garantiza mayor seguridad por control e inclusión social de las comunidades.

Por su parte, la consideración integral y heterogénea de las situaciones existentes a nivel de definición de la política, conlleva necesariamente a desarrollar un abanico amplio de opciones de acceso a un hábitat digno, así como a la utilización y rescate de opciones productivas y constructivas presentes en cada comunidad y territorio. En este sentido, no son los productos sino las modalidades en que éstos se ejecutan lo fundamental, marcando el énfasis que los procesos poseen sobre los productos que puedan ofrecerse o construirse.

El fortalecimiento de estas capacidades locales a nivel del municipio, con el conocimiento y disposición de estrategias y herramientas probadas, resulta así elemental y pertinente, dado que constituyen las bases para el replanteamiento de nuevos modos de definición de políticas públicas, más adecuadas a las necesidades y con mayor reconocimiento de los procesos micropolíticos. De igual manera permiten reposicionar al municipio como actor local estratégico en la consecución del acceso a un hábitat digno en clave trasformadora.

\section{DIMENSIÓN MICROPOLÍTICA DEL HABITAR}

Como hemos señalado anteriormente, es posible pensar los fenómenos de la política más allá -o más acá- de los tradicionales espacios en los que se los piensa (esto es el Estado y sus diferentes institucionalidades). En ese sentido, entendemos que las prácticas sociales que pueden ser conceptualizadas bajo la categoría de "habitar" implican una densidad capaz de tensionar las definiciones consabidas de "hábitat". Ligando estas categorías, hábitat y habitar, a la perspectiva de Deleuze y Guattari podemos pensar los procesos de producción de hábitat en su dimensión micropolítica y así ligar la práctica de habitar como proceso de producción de subjetividad. En ese sentido, coincidimos con la perspectiva propuesta por Vanoli: 
Esto tiene por objetivo aportar elementos que nos permitan comprender a los sujetos desde su hacer y sus prácticas cotidianas, considerándolos como actores claves para aportar a una perspectiva teórico-política del hábitat. La producción de subjetividad es un proceso continuo de formas de hacer, pensar, percibir, afectar, de prácticas que producen y reproducen significados y que encuentran ciertos grados de objetivación en sus representaciones: discursivas, de ordenamiento del territorio, edilicias, tecnológicas, etc (Vanoli, 2018:125-6)

Desde una perspectiva micropolítica, interesa preguntarnos por los efectos de posibilidad que otorgan las intervenciones materiales provistas por el Estado, y abrir allí un cuestionamiento a los mecanismos de recupero de saberes, memorias y sensibilidades propias de las comunidades a las que se dirigen las políticas habitacionales.

Como hemos dejado ver en el apartado anterior, reconocer la articulación entre instancias de diseño e implementación de políticas públicas habilita un espacio de diálogo con las comunidades objeto de estas políticas, entendidos también como actores estratégicos en el diseño, gestión y desarrollo de políticas habitacionales.

En ese sentido, podemos empezar por recuperar conceptualizaciones que profundizan en la dimensión política de la tecnología, señalando la no neutralidad de la misma, y enfoques sociológicos que permiten pensar el diálogo de saberes, como estrategia superadora de enfoques verticalistas, transferencistas, asistencialistas. Bajo esta perspectiva, es necesario determinar -en continuidad con lo propuesto por Winner (1987) y Balabanian (1999)- que las experiencias de la modernidad despegan la idea de neutralidad asociada a la tecnología. Es decir, la tecnología no puede ser comprendida como un simple medio para resolver acciones de la humanidad, por el contrario, es necesario detenerse en la dimensión política de la tecnología, con la necesidad de advertir las innegables definiciones ideológicas y los intereses que guían todo desarrollo o implementación de la misma.

Así, es posible rastrear aquellos procesos de producción de subjetividad antes definidos, actualizados como fenómeno de habitar. Desde allí, podemos visibilizar algunos aspectos extrapersonales, en términos de Deleuze y Guattari, que componen los mecanismos de producción de hábitat, contenidos en las definiciones tecnológicas.

En general, las políticas habitacionales centran su atención en el déficit habitacional, de manera que todo el devenir que se despliega desde esa comprensión, suscitando experiencias subjetivas tendientes a reproducir ese orden: la carencia no es solo material, es también simbólica. Esta comprensión habilita un territorio (cuerpos, proyectos de vida, espacios geográficos) donde 
intervenir por medio de definiciones que no necesariamente son consensuadas con las comunidades receptoras.

Estas definiciones, orientadas a un tipo de producción de subjetividad pasiva, se expresan de manera múltiple. Entre las mismas podemos identificar las tipologías y tecnologías constructivas, las operaciones de gestión territorial, las discursividades en medios de comunicación masiva, los instrumentos de financiación, etc. No obstante, estos actores sociales son capaces, siempre, de realizar una aprehensión singular de estos elementos, los cuales son susceptibles de entramar en los aspectos infrapersonales, antes definidos, compuestos por los horizontes de deseo de los sujetos, inscriptos en sistemas de percepción, memoria, afectos, orgánicos, etc. Esa capacidad de entramar puede ocurrir con mayor o menor grado de conflictividad o avenencia.

Tal como venimos señalando en este artículo, es necesario revisar las premisas desde el origen del planteo, revisar el lugar que ocupa la resolución material (innegable) sin ignorar las posibilidades efectivas que arroja la presencia de saberes, deseos y experiencias por parte de las comunidades a las que el Estado dirige sus acciones. Así, podemos pensar las políticas a través de las cuales el Estado interviene en los territorios, procurando abordar problemáticas habitacionales, como un ejercicio de producción y ponderación de universos de lo posible. Estas intervenciones suponen definiciones que, conscientemente o no, se desdoblan en múltiples niveles: económico, de sociabilización, residencial, etc. Y al mismo tiempo se producen transformaciones en órdenes intangibles, de producción de subjetividad.

Este interjuego entre lo molar y lo molecular, entendido como el proceso de producción de subjetividad siempre tensionado entre las propias pulsiones de singularización y efectos de reproducción del orden dado, encuentran en la producción de hábitat un espacio para agenciar de manera singular. La oportunidad de plasmar en el propio territorio todo aquello que es ponderado en la propia experiencia de vida habilita un plano subjetivo de acceso al hábitat digno.

En ese sentido, y procurando pasar una instancia de articulación de las ideas volcadas hasta aquí, es fértil pensar en procesos de definición del hábitat recuperando experiencias de habitar, es decir, la propia subjetividad de los actores sociales a quienes se dirige la política pública destinada a resolver el acceso al hábitat en procesos de definición participativos y creativos. Boaventura de Sousa Santos (2009) es un autor que puede proveernos de algunas claves pertinentes a estos fines.

Las epistemologías del sur convocan a pensar en un proyecto político y epistémico capaz de subvertir formas vinculares de dominación, principalmente organizadas desde la idea de colonialidad². Diversos autores, plantean diferentes y complementarias dimensiones de la colonialidad; la 
colonialidad del poder (entre ellos Quijano y Grosfoguel) entendida como la interrelación entre formas de explotación y de dominación, la colonialidad del saber (como Lander y Mignolo) vinculada al rol de la epistemología y las tareas de producción de conocimiento en la reproducción de regímenes de pensamiento y la colonialidad del ser (especialmente Maldonado Torres) referida a la experiencia vivida de la colonización y su impacto en el lenguaje.

Entre las distintas categorías que componen este andamiaje conceptual, se recupera aquí la noción de "hybris del punto cero", acuñada por Santiago Castro-Gomez (2007) quien construye esta categoría para describir la mirada colonial sobre el mundo, su modelo epistémico. Entendido como el lugar del comienzo epistemológico, esta categoría permite señalar las operaciones equivalentes al poder de instituir, representar y construir una visión totalizadora de lo real. Como modelo de producción de conocimiento, erige a un observador privilegiado, que se pretende posicionado por fuera del mundo (punto cero) a fin de aplicar sobre él su mirada analítica, aunque pretendidamente orgánica (de ahí hybris, el pecado de la desmesura en la tradición griega). Este modelo epistémico instituye un punto de vista como el punto de vista privilegiado sobre todos los demás puntos de vista posibles, lo cual constituye un aspecto esencial de la epistemología del colonialismo.

Recuperamos esta definición pues comprendemos que en las prácticas de diseño de políticas publicas opera en gran medida este modo vincular, el cual subalterniza o invisibiliza otras aproximaciones a la realidad.

Santos introduce una perspectiva epistemológica que invita a pensar las condiciones de un paradigma de transición, que emerge del reconocimiento de saberes diversos, silenciados por los modos hegemónicos de construcción de saber. La alternativa planteada por el autor implica un tipo de racionalidad orientada a reconocer y recuperar la pluralidad de experiencias que actualmente son desperdiciadas, esto lo sintetiza proponiendo la expansión del presente y la contracción del futuro. El requisito de expandir el presente, denominada Sociología de las Ausencias, requiere de dos momentos, la deconstrucción y la reconstrucción. Deconstruir es aquí un modo de identificar los modos de producir no-existencia, plasmados en las monoculturas del saber, del tiempo lineal, de la clasificación social, de la escala dominante y de la lógica productivista; reconstruir esas realidades negadas implica sustituirlas por cinco ecologías: de los saberes, de las temporalidades, de los reconocimientos, de las transescalas y de las productividades. Contraer el futuro se transforma en un factor de ampliación del presente, y lo señala como un futuro de posibilidades, construidas en el presente, siendo éste el campo de la Sociología de las Emergencias. 
Boaventura de Sousa Santos considera que las alternativas que caben en el horizonte de posibilidades concretas, merecen ser investigadas, tanto en el campo de las experiencias disponibles como el campo de las experiencias sociales posibles; así, la sociología de las ausencias y la sociología de las emergencias se encuentran estrechamente vinculadas. Los procesos de definición de abordajes en el campo del hábitat son espacios donde la multiplicidad y la diversidad se revelan con intensidad: es allí donde recuperar los saberes de quienes habitan, sus experiencias constructivas, sus usos habituales, sus proyecciones de deseo, sus memorias del territorio, sus conflictos vinculares, etc.

La posibilidad de que diversas experiencias, disponibles y posibles, sean puestas en diálogo requiere de un proceso complejo, aquello que el autor denomina traducción. Y es justamente este concepto el que interesa recuperar para la comprensión de los procesos de articulación micro y macropolítica para un abordaje integral en el campo del hábitat. El autor sostiene que la tarea de traducción se propone crear inteligibilidad reciproca entre las experiencias de mundo, por lo que este procedimiento incide tanto sobre los saberes como sobre las prácticas (y sus agentes).

La tarea de traducción implica un trabajo de interpretación entre diferentes experiencias de mundo, con el objetivo de identificar preocupaciones isomórficas y de poner en diálogo las diferentes respuestas que proporcionan (Santos, 2009). El principio heurístico de esta tarea es el de la incompletud de todo saber, y ante el reconocimiento de las propias limitaciones, se abre la posibilidad de un movimiento de apertura al diálogo con otros saberes, también incompletos, pero quizá complementarios en algún sentido. El concepto central sobre el que se sustenta la traducción es el de zona de contacto y se refiere a campos sociales donde diferentes mundos de vida (normativo, prácticas, conocimientos) se encuentran, chocan, interactúan. En una situación concreta de traducción, se define el campo de experiencia que se abordará, a partir de los saberes (y agentes) que entran en diálogo, en interacción. Santos señala además que este diálogo de saberes (interpelación, cuestionamiento, evaluación, etc.) no se produce en una actividad intelectual abstracta, sino en el contexto de prácticas sociales constituidas y/o por constituirse, cuya dimensión epistemológica es una entre otras, y es de esas prácticas que emergen preguntas, espacios de vacancia o fronteras, que permiten articular los saberes en presencia. La superioridad de un saber sobre otro deja de ser definido por el grado de institucionalización y profesionalización, para pasar a ser definida por la contribución pragmática que dicho saber arroja sobre el campo de experiencia que reúne a los agentes. Esta dislocación pragmática de las jerarquías no diluye la asimetría, pero permite nuevas relaciones que se ajustan a un escenario concreto. 
Sobre esa línea de pensamiento entendemos que se abre la posibilidad de recuperar las singularidades con que distintas comunidades constituyen sus modos de habitar, sostenida en memorias, modos de percibir el espacio, modos vinculares, etc. que en definitiva constituyen la dimensión micropolítica aquí desarrollada. A su vez, el rol del Estado como garante de derechos (acceso al suelo, a la vivienda, etc.) puede ser expresado desde diferentes escalas y modalidades institucionales, entre las que destacamos su nivel local por su centralidad, al tratarse de una de las instituciones más cercanas a las realidades de los territorios y por su capacidad potencial de intervención y transformación.

\section{CIERRE DEL TRABAJO: ARTICULACIÓN DE LOS ENFOQUES}

El acceso a la vivienda digna es uno de los derechos que, como ciudadanos argentinos, encontramos definido en la Constitución Nacional (Artículo 14 bis). Este hecho demarca el rol del Estado, en tanto responsable de garantizarlo. No obstante, tal como hemos intentado señalar a lo largo de este artículo, la forma en que se define aquello que es nombrado como "vivienda digna" está dado por un amplio abanico de posturas políticas. Nos interesa especialmente aquellas que cuentan con la activa participación del Estado en tanto garante de derechos y abogamos de manera singular por ejercicios de tal rol que permitan prácticas innovativas, revalorizando ámbitos y saberes locales.

Con lo señalado hasta aquí, intentamos nutrir una perspectiva tendiente a abordar de manera multisituada a los procesos de producción de hábitat. En ese sentido, subrayamos la centralidad que ocupa el recupero de los ámbitos y saberes locales, tanto en instancias de diseño como de implementación de políticas públicas. En ese sentido, consideramos central la institucionalización de espacios y/o estrategias capaces de vehiculizar el diálogo interactoral junto con aquellos factores, saberes y experiencias invisibilizados, a fin de definir aquellas acciones estatales que inciden en el hábitat, de manera integral.

Para ello, dentro de los espacios institucionales (macropolítica) el desarrollo del artículo enfatiza la importancia de recuperar el rol inherente a los gobiernos locales, como dimensión local irrenunciable. En su fortalecimiento resultan claves la promoción de ciertas capacidades como las descriptas, las cuales favorecen la apertura de manera genuina al diálogo con actores de las comunidades, recuperando sus saberes y percepciones (micropolítica). Es esta habilitación de un espacio de interjuego molar-molecular entre ambos niveles, la que es capaz de producir aportes 
sustantivos a la comprensión y resolución de las problemáticas de hábitat en perspectiva transformadora.

La necesidad de consideración y fortalecimiento local se encuentra relacionada a su vez al imperioso crecimiento democrático y participativo de la ciudadanía, quien debería encontrar (y reclamar), en el espacio local, el lugar privilegiado para ejercer sus derechos y obligaciones. Este proceso encuentra sus posibilidades de atención y resolución no sólo en el impulso de políticas que incluyan a los municipios y a las comunidades receptoras como sujetos activos en su gestión e implementación, sino en estrecha recuperación de las capacidades de dichos actores, en cuanto sujetos factibles de recuperar el rol que les compete en materia urbano-territorial (Cepal-MinurviNU, 2016 y Autora, año).

Desde la perspectiva abordada, estas capacidades se encuentran particularmente relacionadas al diálogo de saberes con la comunidad y con otros actores locales presentes en el territorio, al que se deberían orientar las prácticas estatales. A la luz de los aportes de Boaventura de Sousa Santos, supone visibilizar las formas de saber o emergentes en el territorio no gestados bajo formas hegemónicas a partir de la promoción de una perspectiva democrática de producción del conocimiento, orientado al desarrollo de tecnologías para una real inclusión social en el campo del hábitat, cuyo centro no sea únicamente la producción material sino la promoción de una dimensión relacional y epistémica diferente a partir de ellas (Autora, año).

La complementariedad entre los niveles macro y micro -desarrollado en el artículo a partir de los aportes político-filosóficos de Félix Guattari y Gilles Deleuze-, puede ser abordado desde el enfoque de diálogo de saberes también presentado. Esto cristaliza en una perspectiva de análisis e intervención territorial/local, capaz de integrar niveles de mayor concreción, como pueden ser las propias organizaciones y sus normativas, hasta los niveles más intangibles como puede ser el proceso de construcción de subjetividad, entendida como aquello que compone las matrices de sentido de los sujetos: mecanismos de percepción, de afectividad, de producción de conocimiento, de memoria, etc.

En ese sentido, el esquema macropolítico convencional de abordaje a la problemática de hábitat debiera integrar la dimensión municipal y productiva del hábitat. La transformación de tal escenario inicial habilita procesos de integración entre organizaciones locales, otorgando sinergia entre la instancia de reconocimiento de las necesidades y capacidades diversas, la productiva y la generación de tecnologías constructivas. En ese plano se constata que el abordaje de problemáticas o demandas vinculadas al hábitat requiere de dinámicas interactorales, como trama que debe hacer sentido a 
nivel local. Es decir, no se trata de la imposición de diagnósticos, prácticas y procesos extralocalmente predefinidos, sino que es necesario el proceso de definición local. Estudios como el de Thomas (2011), respecto de la implementación de tecnología social, el de Graglia (2004) y Rodulfo (2005), relacionados a políticas públicas y a modelos de gestión relacional o Autora (año), remitiendo al diseño e implementación modelos de gestión innovadores en el campo del hábitat son referencias a la hora de pensar en dinámicas y prácticas alternativas.

Así, el impulso de una revalorización de los ámbitos y saberes locales en el campo habitacional tiene, al menos un cuádruple propósito. En primer lugar, señalar la imposibilidad de asimilar el abordaje de problemáticas de hábitat a la implantación de un objeto arquitectónico, ya que éste es únicamente la expresión material de un proceso multidimensional. En segundo lugar, enfatizar que el ámbito y actores participantes en las fases de diseño de la política no resultan indiferentes sino, por el contrario, claramente estructurantes. En tercero, renunciar a la idea de neutralidad de la tecnología, abriendo la "caja negra" de ese proceso multidimensional antes señalado. Y en cuarto, establecer que la recuperación de la dimensión local de la macropolítica -con su consecuente potenciación de capacidades específicas- resulta ineludible a la hora de recuperar los procesos micropolíticos, evidenciando la interinfluencia entre las dimensiones macro y micro.

Sin que estos propósitos sean reconocidos y puestos en desarrollo, toda intención trasformadora e innovativa no superará el cambio circunstancial y específico, impidiendo la real resolución de las problemáticas socio-habitacionales vigentes.

\footnotetext{
${ }^{1}$ Particularmente en este texto haremos referencia a dos obras en las que se recogen aportes significativos de los autores: "Mil mesetas. Capitalismo y esquizofrenia" (Deleuze, G. y Guattari, F., 1997) y "Micropolítica: cartografías del deseo" (Guattari, F. y Rolnik, S., 2013)

${ }^{2}$ La noción de colonialismo implica una relación política y económica en el marco de la cual la soberanía de un pueblo queda supeditada a otro. Autores como Santiago Castro-Gómez y Ramón Grosfoguel, señalan que "la división internacional del trabajo entre centros y periferias, así como la jerarquización étnico-racial de las poblaciones, formada durante varios siglos de expansión colonial europea, no se transformó significativamente con el fin del colonialismo y la formación de los Estados-nación en la periferia. Los autores señalan que se asiste, más bien, a una transición del colonialismo moderno a la colonialidad global, proceso que ciertamente ha transformado las formas de dominación desplegadas por la modernidad, pero no la estructura de las relaciones centro-periferia a escala mundial". Desde el enfoque 'decolonial', "el capitalismo global contemporáneo resignifica (...) las exclusiones provocadas por las jerarquías epistémicas, espirituales, raciales/étnicas y de género/sexualidad desplegadas por la modernidad" (Castro-Gómez y Grosfoguel, 2007:13-14).
} 


\section{BIBLIOGRAFÍA CITADA.}

ALCALÁ, Luis Cortés (1995). "La vivienda como objeto de comprensión”. En: Alcalá Luis Cortés (Comp.) (1995) Pensar la vivienda. Ed. Agora. Talasa Ediciones S. L. Madrid, España.

AROCENA, José (1995) El desarrollo local: un desafío contemporáneo. Ed. Nueva Sociedad, CLAEH y Universidad Católica del Uruguay. Caracas, Venezuela. Disponible en:

http://200.41.82.27/cite/media/2016/02/Arocena-J_2002_El-desarrollo-local-un-desafiocontemporaneo-Capitulo-I.pdf (consulta: 21/01/2019)

BALABANIAN, Norman (1999). "La tecnología, ¿ama o esclava?". En Revista Llull, revista de la sociedad española de historia de las ciencias y de las técnicas, $n^{\circ} 43$, pp 22, 5-36. Zaragoza: Universidad de Zaragoza

BRUGUÉ, Joaquim y GOMÁ, Ricard (1998), Gobiernos locales y políticas públicas. Bienestar social, promoción económica y territorio, Ariel Ciencia Política, Barcelona, España.

BARRETO, Miguel Ángel y ALCALÁ, Laura Inés (2007) “Cambios en las Prestaciones urbanoambientales de la política habitacional Argentina"- Reflexiones a partir de cuatro programas orientados a población en situación de pobreza del Gran Resistencia" en: Pipa, D., Peyloubet, P. De Salvo, L. (2008) Ciencia y tecnología para el hábitat popular. Desarrollo tecnológico alternativo para la producción social del hábitat. Editora Nobuko. Buenos Aires- Argentina.

CASTRO-GOMEZ, Santiago y GROSFOGUEL, Ramón (2007). "Prólogo. Giro decolonial, teoría crítica y pensamiento heterárquico", en El giro decolonial: reflexiones para una diversidad epistémica más allá del capitalismo global, compiladores Santiago Castro-Gómez y Ramón Grosfoguel, Bogotá, Siglo del Hombre Editores, Universidad Central, Instituto de Estudios Sociales Contemporáneos y Pontificia Universidad Javeriana, Instituto Pensar

CASTRO-GOMEZ, Santiago (2007) “Decolonizar la universidad. La hybris del punto cero y el diálogo de saberes", en El giro decolonial: reflexiones para una diversidad epistémica más allá del capitalismo global, compiladores Santiago Castro-Gómez y Ramón Grosfoguel, Bogotá, Siglo del Hombre Editores, Universidad Central, Instituto de Estudios Sociales Contemporáneos y Pontificia Universidad Javeriana, Instituto Pensar.

AUTORA, Libro

CEPAL-MINURVI-NU (Comisión Económica para América Latina y el Caribe-Foro de Ministros y Autoridades Máximas de la Vivienda y el Urbanismo de América Latina y el Caribe-Naciones Unidas) (2016) América Latina y el Caribe. Desafíos, dilemas y compromisos de una agenda urbana común. CEPAL, Santiago de Chile, Chile. Disponible en: http://www.cepal.org/es/publicaciones/40656america-latina-caribe-desafios-dilemas-compromisos-agenda-urbana-comun (consulta: 22/02/2017).

CRAVACUORE, Daniel (2014) "La recentralización emergente en América Latina” En: Fidel, C. y Villar, A. (comp) (2014) Miradas y controversias del desarrollo territorial en Argentina: aproximación a un enfoque analítico. Ediciones del CCC Centro Cultural de la Cooperación Floreal Gorini. Universidad Nacional de Quilmes. Bernal, Ciudad Autónoma de Buenos Aires, Argentina.

CRAVACUORE, Daniel (2015) "Recentralización municipal en América Latina. Algunas razones explicativas". Ponencia presentada en la XI Conferencia Interamericana de Alcaldes. Miami, EE.UU.

DE SOUSA SANTOS, Boaventura (2009) Una epistemología del sur: la reivindicación del conocimiento y la emancipación social. México: Siglo XXI. CLACSO.

DELEUZE, Gilles y GUATTARI, Félix (1997). Mil Mesetas. Capitalismo y esquizofrenia. Valencia: PreTextos. 
AUTORA, Libro.

AUTORA, Libro.

AUTORA, Libro.

AUTORA, Libro.

AUTORA Libro.

GRAGLIA, Emilio (2004) Diseño y gestión de políticas públicas hacia un modelo relacional. Serie PROFIM Volumen 1. EDUCC Editorial de la Universidad Católica de Córdoba. Córdoba, Argentina

GLOBAL TRASKFORCE (2016) Declaración de la Segunda asamblea mundial de gobiernos locales y regionales en la Tercera Conferencia de la ONU sobre vivienda y desarrollo urbano sostenible-Hábitat III. The Global Network of Cities, Local and Regional Governments, Quito, Ecuador. Diponible en: https://www.uclg.org/sites/default/files/declaracion_de_la_2a_asamblea_mundial_a_habitat_iii.p df (consulta: 24/02/2017).

GONZALÉZ MEYER, Raúl (2009) "Lo local en la teoría y en la política" En: Polis, Revista de la Universidad Bolivariana, Volumen 8, № 22, 2009, p. 67-80. Disponible en:

https://scielo.conicyt.cl/pdf/polis/v8n22/art05.pdf (consulta: 21/01/2019)

GRÉMION, Pierre (1976), Le pouvoir périphérique, Edition du Seuil, Paris.

GUATTARI, Félix y ROLNIK, Suely (2013). Micropolítica: cartografías del deseo. Buenos Aires: Tinta Limón

AUTORA, Libro.

NACIONES UNIDAS (2016) Nueva Agenda Urbana. Resolución aprobada por la Asamblea General el 23 de diciembre de 2016. ONU Hábitat, Quito, Ecuador. Disponible en: http://habitat3.org/wpcontent/ uploads/New-Urban-Agenda-GA-Adopted-68th-Plenary-N1646660-S.pdf (consulta: 24/02/2017).

NIRENBERG, Olga, BRAWERMAN, Josette y RUIZ, Violeta (2006) Programación y evaluación de proyectos sociales. Aportes para la racionalidad y la transparencia. Paidós. Buenos Aires, Argentina.

PEDROTTI, Carolina (2016), Calidad residencial y condiciones de producción en la vivienda social promovida por el sector privado. Zona Metropolitana de Toluca, 2001-2011, Programa Universitario de Estudios sobre la Ciudad/UNAM-Instituto del Fondo Nacional de la Vivienda para los Trabajadores, Ciudad de México, México.

PETRELLI, Hernán, GALIZZI, Daniel y ORECCHIA, Mariana (2014) “Capacidades estatales de intervención territorial", en: Instituto Provincial de Administración Pública (IPAP). Líneas de investigación 2014. Informe final. Ed. Propia. La Plata, Argentina.

RAMÍREZ, Ronaldo (2002). "Evaluación social de políticas y programas de vivienda: un análisis de la contribución de la vivienda a la reducción de la pobreza urbana". En: Boletín del Instituto de la Vivienda (INVI). Volumen 17. Número 045. Mayo de 2002. Universidad de Chile. Santiago, Chile.

REESE, Eduardo (2003) Instrumentos de gestión urbana, fortalecimiento del rol del municipio y desarrollo con equidad. Material de estudio del Curso de Gestión Urbana. Lincoln Institute. 9 al 19 de febrero de 2003. Lima, Perú.

RODRÍGUEZ, Marcela, TABORDA, Alberto, EULA, Mónica, CAMISASSO, Mónica, GUGLIELMELLI WHITE, Ana (2011) La evaluación de las políticas habitacionales. Significados y estados del arte. Cuadernos de trabajo. Serie Investigación N.․ 1. Escuela de Trabajo Social, Universidad Nacional de Córdoba. Córdoba, Argentina 
RODULFO, María Beatriz (2005) La evolución de la gestión en políticas habitacionales y su relación con el desarrollo local. El caso argentino. Análisis comparativo de modelos de gestión. Exposición en el XI Encuentro de la Red ULACAV: UNIVERSIDAD Y VIVIENDA SOCIAL-Experiencias Académicas para la Gestión Local del Hábitat. Rosario, Argentina.

SAUTU, Ruth; BONIOLO, Paula; DALLE, Pablo y ELBERT, Rodolfo (2005) Manual de metodología. Buenos Aires: Clacso

SUBIRATS, Joan, KNOEPFEL, Peter, LARRUE, Corinne y VARONNE, Frederic (2008) Análisis y gestión de políticas públicas. Ariel Ciencia Política. Barcelona, España.

THOMAS, Hernán (2011) "Tecnologías para la inclusión social y políticas públicas en América Latina". Ponencia presentada en el II Workshop Internacional sobre Tecnologia Social e Políticas na América Latina en el mes de junio de 2011. Universidad Estatal de Campinas (UNICAMP), Campinas, Brasil.

THWAITES REY, Mabel (2010). Después de la globalización neoliberal. ¿Qué Estado en América Latina? Año XI, 27, 19-44. Argentina: OSAL. Disponible en http://www.cronicon.net/paginas/Documentos/Despu\%E9s\%20de\%20la\%20glob\%20neoliberal.pdf

UCLG-CGLU (Red Mundial de Ciudades y Gobiernos Locales y Regionales-United Cities and Local Governments) (2016) El compromiso de Bogotá y la agenda de acción. Declaración del $5^{\circ}$ Congreso de CGLU. Cumbre Mundial de Líderes Locales y Regionales, 15 octubre, Bogotá, Colombia. Disponible en: https://www.uclg. org/sites/default/files/el_compromiso_de_bogota_0.pdf (consulta: 24/02/2017).

VANOLI, Fernando (2018) "Hábitat como campo de disputa: agenciamientos colectivos ante un sistema de dominación múltiple”. En revista Cuaderno Urbano n²4, pp. 117-136. Chaco: Universidad Nacional del Noroeste.

WINNER, Langdon. (1987). La ballena y el reactor. Una búsqueda de los límites en la era de la alta tecnología. (Edición revisada y anotada de Javier Bustamante). Barcelona: Gedisa.

ZICCARDI, Alicia y CRAVACUORE, Daniel (2017) Los gobiernos locales y las políticas de vivienda en México y América Latina. UNAM. México.

Tabalho enviado em 29 de abril de 2019

Aceito 02 de fevereiro de 2020 